\title{
Ramos colaterais do arco aórtico de Callithrix penicillata (É. Geoffroy, 1812) (Primates, Cebidae)
}

\author{
José Ricardo Nascimento de Souza Neto \\ Ana Rita de Lima \\ Érika Branco * \\ Laboratório de Pesquisa Morfológica Animal, Instituto da Saúde e Produção Animal \\ Universidade Federal Rural da Amazônia, Campus de Belém \\ Avenida Presidente Tancredo Neves, 2.501, CEP 66077-830, Belém - PA, Brasil \\ * Autor para correspondência \\ ebranco.ufra@gmail.com
}

Submetido em 21/09/2018

Aceito para publicação em 23/01/2019

\section{Resumo}

Callithrix penicillata é um primata não humano endêmico no Brasil, amplamente utilizado para estudos biomédicos, porém, pouco se conhece quanto à anatomia desses indivíduos, incluindo aspectos vasculares. Assim, objetivamos descrever o arco aórtico dessa espécie, a fim de corroborar tanto estudos comparados quanto ensaios hemodinâmicos para modelos experimentais. Foram avaliados cinco C. penicillata, que apresentaram dois tipos distintos de angioarquitetura do arco aórtico, sendo um comumente encontrado em outros mamíferos, e outro em similaridade com Saguinus niger, sugerindo que tal padrão possa ter relação com o fato de ambas as espécies pertencerem à Família Callithrichidae.

Palavras-chave: Anatomia comparada; Angioarquitetura; Sagui-de-tufo-preto; Vascularização arterial

\section{Abstract}

Collateral branches of the aortic arch of Callithrix penicillata (É Geoffroy, 1812) (Primates, Cebidae). Callithrix penicillata is a non-human primate endemic to Brazil. It is widely used in biomedical studies; however, little is known about the anatomy of this species, including the vascular aspects. Therefore, this work describes the aortic arch of this species, in order to corroborate comparative studies and hemodynamic tests for experimental models. Five $C$. penicillata were evaluated, which presented two distinct types of aortic arch angioarchitecture, one commonly found in other mammals, and another similar to Saguinus niger, suggesting that this pattern may be related to the fact of both species belong to the family Callithrichidae.

Key words: Angioarchitecture; Arterial vascularization; Black-tufted-ear marmoset; Comparative anatomy 


\section{Introdução}

A espécie Callithrix penicillata, conhecida como sagui-de-tufo-preto ou mico-estrela, é um mamífero da Ordem Primata, amplamente distribuído em florestas de galeria no Cerrado brasileiro e Mata Atlântica; com atividade diurna, pesando em média $350 \mathrm{~g}$; apresenta uma cauda que chega a medir $32 \mathrm{~cm}$, sendo maior que seu corpo, com cerca de 20 a $22 \mathrm{~cm}$. Vivem cerca de 10 anos em vida livre e podem chegar aos 16 anos em cativeiro, atingindo maturidade para cópula aos 18 meses (FARIA, 1989; AURICCHIO, 1995; NOWAK, 1999). Geralmente são encontrados em bandos de 4 a 15 animais (RYLANDS; MENDES, 2018) e alimentam-se de filhotes e ovos de pássaros, diversos insetos, pequenos lagartos, frutos, néctar e exsudatos de plantas, sendo considerado um animal onívoro (AURICCHIO, 1995; BICCAMARQUES et al., 2006).

Assim como outros saguis do gênero Callithrix, possui uma pelagem estriada no dorso e na cauda, apresentando tufos de pelos nas orelhas. A presença dos ornamentos pré-auriculares, longos e negros em forma de pincel, assegura-lhe o nome de sagui-de-tufopreto e os diferenciam de outras espécies do gênero (AURICCHIO, 1995; BICCAMARQUES et al., 2006). Tais espécies possuem uma grande importância na área da pesquisa biomédica, não somente por sua ocorrência nos ecossistemas da América Latina, mas também devido seu pequeno porte, facilitando seu manejo, biossegurança e particularidades fisiológicas, como sua alta taxa de fecundidade (STEVENSON; RYLANDS, 1988; ABBOTT et al., 2003; MANSFIELD, 2003; VALLE, 2007).

Apesar do interesse nessa espécie para pesquisas biomédicas, a morfologia desses indivíduos ainda é pouco explorada, com registros literários relacionados apenas com partes dos sistemas digestório (SILVA, 2012; NITTA et al., 2013), circulatório (SILVA et al., 2012a), respiratório (SILVA et al., 2012b) e sistema nervoso (SANTOS et al., 2016). Entretanto, não existem registros quanto ao estudo da vascularização no $C$. penicillata, principalmente no que tange à principal artéria do corpo, a aorta, que emerge do ventrículo esquerdo, medialmente ao tronco pulmonar. Após seu surgimento, a aorta estende-se cranialmente, coberta pelo pericárdio, e curva-se dorsalmente para a esquerda formando um arco (SCHALLER; NASCIMENTO, 1999; FURTADO et al., 2017).

Do arco aórtico surgem ramos para a cabeça e membros torácicos, destacando o tronco braquiocefálico e artéria subclávia esquerda, porém, ocorrem variações entre as espécies, podendo originar outros vasos (MOORE; DALLEY, 2001; TORTORA, 2007; ITEREZOTE et al., 2009). Frente a essa possibilidade decidimos investigar a disposição dos ramos colaterais do arco aórtico do C. penicillata, bem como suas ramificações principais, visando subsidiar não apenas novos estudos comparativos, mas bem alicerçar pesquisas hemodinâmicas.

\section{Material e Métodos}

Este estudo está de acordo com as normas preconizadas pelo Comitê de Pesquisas com Animais (CEPAN/IEC/SVS/MS), № 008/2010.

Foram avaliados cinco animais jovens, sendo quatro machos e uma fêmea, provenientes do Centro Nacional de Primatas CENP - Ananindeua, PA, que foram a óbito por causas naturais, congelados e encaminhados ao Laboratório de Pesquisa Morfológica Animal (LaPMA), da Universidade Federal Rural da Amazônia (UFRA).

Inicialmente, foi dissecada a aorta torácica, com abordagem pelo antímero esquerdo após remoção de todas as costelas do respectivo antímero em detrimento do diminuto tamanho do animal. $\mathrm{O}$ vaso foi canulado nas direções cranial e caudal e foi injetado aproximadamente $10 \mathrm{~mL}$ de látex Neoprene 450 contrastado com sulfato de bário, corado com pigmento vermelho, seguido de fixação com solução de formol a $10 \%$ pelas vias intramuscular e intracavitária. Posteriormente, o vaso foi mantido submerso, na mesma solução fixadora, em recipiente adequado por sete dias, seguido de dissecação dos ramos colaterais do arco aórtico da espécie, fotodocumentação e realização radiografias torácicas, nas quais os animais ficaram na posição ventrodorsal com tórax aberto. Em seguida o esterno foi removido para melhor visualização da cavidade torácica, favorecendo a dissecação e identificação dos vasos. 
A nomenclatura adotada foi baseada na Nomenclatura Anatômica Veterinária ilustrada (INTERNATIONAL COMMITTEE ON VETERINARY GROSS ANATOMICAL NOMENCLATURE, 2017).

\section{Resultados}

Os ramos originados do arco aórtico do $C$. penicillata apresentaram duas configurações diferentes, no qual em dois machos e na fêmea houve a formação de três artérias, sendo elas o tronco braquiocefálico, carótida comum esquerda e subclávia esquerda.
Destacamos que, em decorrência da remoção do esterno, não foi possível isolar as artérias torácicas internas.

Do tronco braquiocefálico (primeiro ramo do arco aórtico) surgiram as artérias carótida comum direita e subclávia direita, e a partir desta última ramificaram-se: artéria vertebral direita, tronco costocervical direito e artéria cervical superficial direita com seu ramo axilar.

O segundo ramo do arco aórtico compreendeu a artéria carótida comum esquerda e, imediatamente, como terceiro ramo, emergiu a artéria subclávia esquerda. Esta última também emitiu, na mesma sequência, as mesmas artérias de sua homóloga contralateral (Figuras 1A; B).

FIGURA 1: A - Radiografia torácica do arco aórtico e seus ramos colaterais em Callithrix penicillata, na posição ventrodorsal, o qual emitiu três ramos diretamente do arco: a - arco aórtico; b - artéria carótida comum direita; $\mathrm{c}$ - artéria carótida comum esquerda; $\mathrm{d}$ - artéria subclávia esquerda; $\mathrm{e}$ - artéria subclávia direita; $\mathrm{f}$ - artéria vertebral esquerda; $\mathrm{g}$ - artéria vertebral direita; $\mathrm{h}$ - tronco costocervical esquerdo; $\mathrm{i}$ - tronco costocervical direito; $\mathrm{j}$ - artéria cervical superficial esquerda; $\mathrm{k}$ - artéria cervical superficial direita; 1 - artéria axilar esquerda; $\mathrm{m}$ - artéria axilar direita. Observar que, neste padrão vascular arterial, o tronco braquiocefálico (*) origina as artérias carótida comum direita (b) e subclávia direita (e). B - Fotomacrografia, em vista ventral, da cavidade torácica de um espécime de Callithrix penicillata observando a ramificação do arco aórtico: a - arco aórtico; b - artéria carótida comum direita; c - artéria carótida comum esquerda; d - artéria subclávia esquerda; e - artéria subclávia direita; f - artéria vertebral esquerda; $g$ - artéria vertebral direita; $\mathrm{h}$ - tronco costocervical esquerdo; $\mathrm{i}$ - tronco costocervical direito; $\mathrm{j}$ - artéria cervical superficial esquerda; $\mathrm{k}$ - artéria cervical superficial direita; 1 - artéria axilar esquerda; $\mathrm{m}$ - artéria axilar direita. Observar que, neste padrão vascular arterial, o tronco braquiocefálico $(*)$ origina as artérias carótida comum direita (b) e subclávia direita (e).
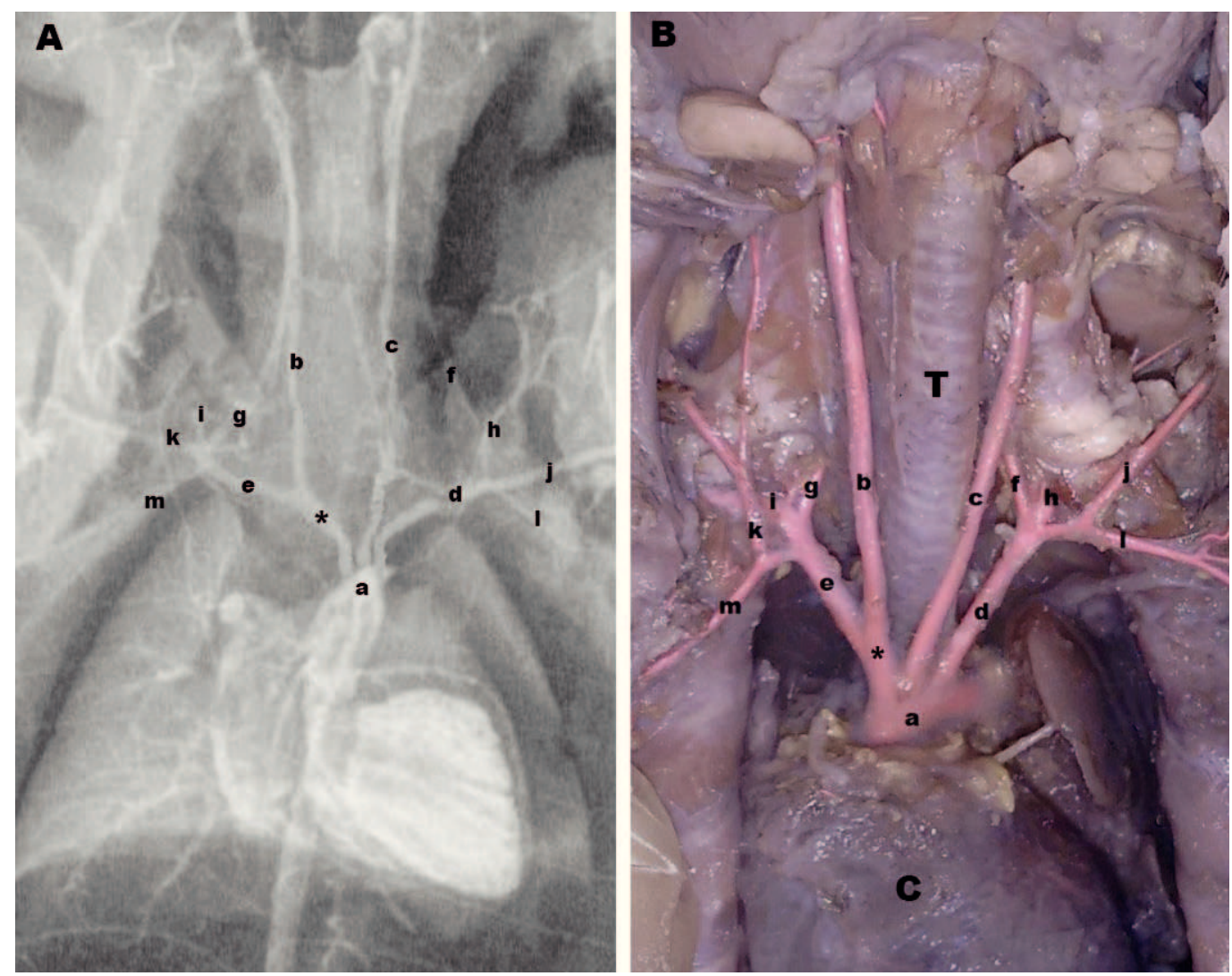
Nos dois outros machos estudados, foram emitidos do arco aórtico apenas o tronco braquiocefálico e a artéria subclávia esquerda. Do tronco braquiocefálico houve a emissão de um tronco comum, que se ramificou nas artérias carótida comum direita e subclávia direita, e a artéria carótida comum esquerda. As artérias subclávias obedeceram ao mesmo padrão de ramificação como já descrito nos espécimes que tiveram três ramos emitidos diretamente do arco aórtico (Figura 2 A e B).

\section{Discussão}

Devido à escassez literária sobre a ramificação do arco aórtico em primatas não humanos, pautamos nossa discussão não apenas em animais dessa Ordem, mas os comparamos com outros mamíferos que julgamos pertinente, observando que a arquitetura do arco aórtico do $C$. penicillata encontra-se numa condição intermediária.

FIGURA 2: A - Radiografia torácica do arco aórtico e seus ramos colaterais em Callithrix penicillata, na posição ventrodorsal, o qual emitiu dois ramos diretamente do arco: a - arco aórtico; b - artéria subclávia esquerda; c - artéria carótida comum esquerda; $\mathrm{d}$ - artéria carótida comum direita; $\mathrm{e}$ - artéria subclávia direita; $\mathrm{f}$ - artéria axilar direita; $\mathrm{g}$ - artéria cervical superficial direita; $\mathrm{h}$ - tronco costocervical direito; $\mathrm{i}$ - artéria vertebral direita; $\mathrm{j}$ - artéria costocervical esquerda; $\mathrm{k}$ - artéria axilar esquerda; 1 - artéria vertebral esquerda; $\mathrm{m}$ - artéria cervical superficial esquerda. Observar que, neste padrão vascular arterial, o tronco braquiocefálico $(*)$ origina as artérias carótida comum direita (d) e carótida comum esquerda (c). B - Fotomacrografia, em vista ventral, da cavidade torácica de Callithrix penicillata observando a ramificação do arco aórtico: a - arco aórtico; b artéria subclávia esquerda; c - artéria carótida comum esquerda; d - artéria carótida comum direita; e - artéria subclávia direita; f - artéria axilar direita; $\mathrm{g}$ - artéria cervical superficial direita; $\mathrm{h}$ - tronco costocervical direito; $\mathrm{i}$ - artéria vertebral direita; $\mathrm{j}$ - artéria costocervical esquerda; $\mathrm{k}$ - artéria axilar esquerda; 1 - artéria vertebral esquerda; $\mathrm{m}$ - artéria cervical superficial esquerda. Observar que, neste padrão vascular arterial, o tronco braquiocefálico (*) origina as artérias carótida comum direita (d) e carótida comum esquerda (c).
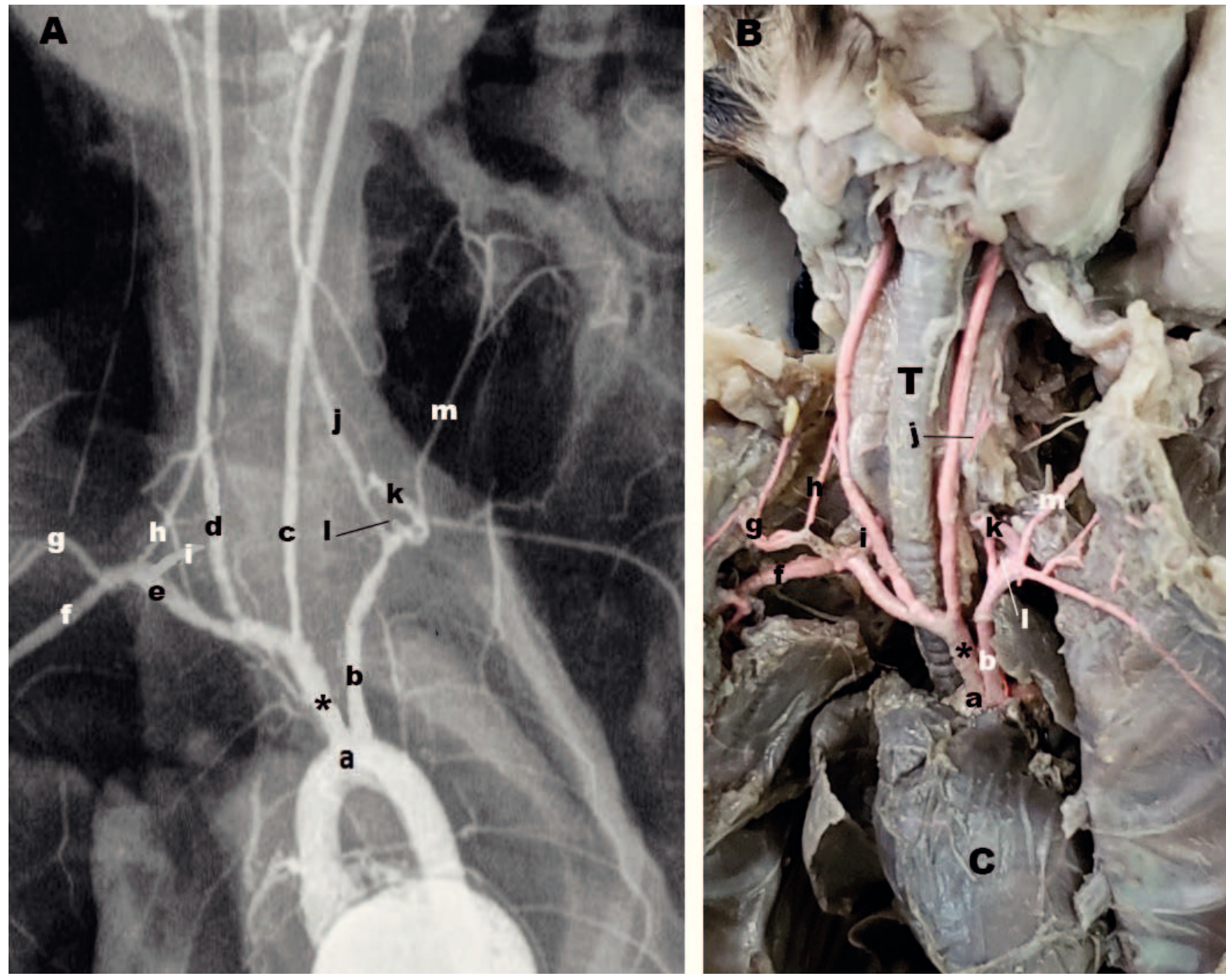
Inicialmente evidenciamos a existência de padrões anatômicos diferentes do arco aórtico em C. penicillata, fato já aludido por Silva et al. (2012) em humanos, no qual os autores relatam quatro padrões anatômicos de arco aórtico e suas ramificações (A, B, C e D), sendo que, dos 19 arcos aórticos analisados, 15 apresentaram o padrão considerado comum, ou tipo A, em que o arco deu origem aos ramos: tronco braquiocefálico, artéria carótida comum esquerda e artéria subclávia esquerda, assim como constatado em três $C$. penicillata analisados. Os autores relatam ainda que em apenas um humano foi encontrado um arco aórtico do tipo $\mathrm{C}$, ou seja, com apenas dois ramos colaterais emergindo, sendo eles o tronco braquiocefálico e a artéria subclávia esquerda. Este padrão também foi achado em $C$. penicillata, nos dois animais estudados.

Assim como no C. penicillata, no Saguinus niger (BRANCO et al., 2017) e no Sapajus apella (FURTADO et al., 2017) também foram observadas as mesmas variações quanto à disposição dos ramos do arco aórtico, ou seja, arco classificado como tipo A. No entanto, Branco et al. (2017) também relataram a presença de arco aórtico tipo C em $S$. niger, ou seja, essa espécie, assim como o $C$. penicillata, também possui os mesmos tipos de classificação do arco aórtico (tipos A e C). Além disso, as ramificações das subclávias direita e esquerda foram as mesmas no $S$. niger, $S$. apella e no C. penicillata, nos quais esses ramos deram origem às artérias vertebral, costocervical, cervical superficial e axilar, cada qual com sua nomenclatura final de acordo com seu antímero. E devido à similaridade da estruturação do arco aórtico entre esses animais, levamo-nos a questionar se poderia ser essa uma característica dos animais da Família Callithrichidae, tendo em vista que ambas as espécies pertencem a esse grupo (SILVA et al., 2017).

Avaliando a literatura sobre o arco aórtico de outros grupos de mamíferos, com maior proximidade filogenética com os primatas, começamos pela Ordem Lagomorpha, na qual coelhos da raça Nova Zelândia (Oryctolagus cuniculus) apresentam variações do arco aórtico, com uma angioarquitetura predominantemente classificada como sendo do tipo $\mathrm{C}$, ou seja, o arco aórtico do $O$. cuniculus tem essencialmente como ramos primários o tronco braquiocefálico e a artéria subclávia esquerda, assim como dois dos C. penicillata estudados. Entretanto, outros $O$. cuniculus apresentaram classificação do tipo A, revelando tronco braquiocefálico, artéria carótida comum esquerda e artéria subclávia esquerda como ramos colaterais do arco aórtico igualmente a três indivíduos de $C$. penicillata. Porém, nos coelhos da Nova Zelândia predominaram como ramos da artéria subclávia direita, de medial para lateral, as artérias: vertebral como primeiro ramo, tronco comum cervical-superficial profundo como segundo e tronco costocervical-torácico interno em terceiro. Já pela artéria subclávia esquerda, predominaram como ramificações, de medial para lateral, as artérias: vertebral, como primeiro ramo, e intercostal suprema seguida da torácica interna (SOUZA et al., 2013), sendo que essas ramificações das subclávias são diferentes das encontradas nos micos-estrelas.

Além da Ordem Lagomorpha, observamos os registros literários da Ordem Rodentia, a qual também apresentou o padrão tipo $\mathrm{C}$ de arco aórtico em Agouti paca (OLIVEIRA et al., 2001), Chinchilla lanigera (ARAÚJO et al., 2004), Kerodon rupestres (MAGALHÃES et al., 2007) e Cavia aperea (OLIVEIRA et al., 2015). No entanto, quando analisamos a Hydrochoerus hydrochaeris, a arquitetura do arco aórtico se mostrou completamente diferente não somente dos roedores aqui comparados, como dos mamíferos em geral citados, uma vez que do arco aórtico surge apenas o tronco braquiocefálico que origina todas as demais artérias que nutrem o pescoço, a cabeça e o membro torácico (CULAU et al., 2007).

Avançando para uma Ordem de mamíferos maiores, temos o Leopardus pardalis (MARTINS et al., 2010), da Ordem Carnivora, que possuiu o mesmo padrão de arco aórtico que dois C. penicillata, no qual houve a emissão de dois ramos colaterais do arco aórtico (tronco braquiocefálico e artéria subclávia esquerda), mantendo o padrão até então identificado nesta revisão, como sendo o tipo C. Além disso, na jaguatirica houve a emissão de um tronco comum entre as artérias carótida comum direita e subclávia direita, assim como visualizado nos dois espécimes de sagui-de-tufo-preto supramencionados. A literatura ainda revela que alguns animais da mesma Ordem do L. pardalis, como o 
gato doméstico (FERNANDES FILHO; BORELLI, 1970), o Procyon cancrivorus (SANTOS et al., 2004) e o Cerdocyon thous (LIMA et al., 2016), também apresentaram como ramos colaterais do arco aórtico o tronco braquiocefálico e a artéria subclávia esquerda, estando estes dentro do mesmo grupo, tipo C.

Por fim, e já um pouco mais distante da Ordem Primata, identificamos registros de que na Ordem Pilosa, mais precisamente em Tamandua tetradactyla (PINHEIRO et al., 2012), assim como na Ordem Marsupialia, em Didelphis albiventris (SCHIMMING et al., 2016), o arco aórtico desses indivíduos também apresenta o mesmo comportamento quanto a sua arquitetura, em relação ao demais mamíferos citados, ou seja, padrão tipo $\mathrm{C}$.

Diante dos achados, concluímos que o arco aórtico de $C$. penicillata revelou comportamento similar ao $S$. niger, ou seja, arcos aórticos classificados como tipo A e C, porém, não podemos afirmar que esse é um padrão dos indivíduos da Família Callithrichidae, pois mais espécies desse grupo precisariam ser avaliadas.

Entre os demais mamíferos aqui comparados, somente o Sapajus apella e alguns Oryctolagus cuniculus apresentaram arco aórtico tipo A, à semelhança também de alguns indivíduos de C. penicillata.

Todos os demais mamíferos já analisados e aqui registrados mantêm o padrão tipo $\mathrm{C}$, assim como parte dos C. penicillata estudados.

\section{Referências}

ABBOTT, D. H.; BARNETT, D. K.; COLMAN, R. J.; YAMAMOTO, M. E.; SCHULTZ-DARKEN, N. J. Aspects of common Marmoset basic biology and life history important for biomedical research. Comparative Medicine, Memphis, v. 53, n. 4, p. 339-350, 2003.

ARAÚJO, A. C. P.; OLIVEIRA, J. C. D.; CAMPOS, R. Ramos colaterais do arco aórtico e as suas principais ramificações em chinchilas (Chinchilla lanigera). Revista Portuguesa de Ciências Veterinárias, Lisboa, v. 99, p. 53-58, 2004.

AURICCHIO, P. Primatas do Brasil. São Paulo: Terra Brasilis, 1995. $168 \mathrm{p}$.

BICCAMARQUES, J. C.; SILVA, V. M.; GOMES, D. F. Ordem Primates. In: REIS, N. R.; PERACCHI, A. L.; PEDRO, W. A.; LIMA, I. P. (Ed.). Mamíferos do Brasil. Londrina: Universidade Estadual de Londrina, 2006. p. 101-148.
BRANCO, E.; CRUZ DE ARAGÃO, M. B.; MARTINS, D. M.; COSTA DO CARMO, D.; TEIXEIRA, J. S.; DE LIMA, A. R. $\mathrm{O}$ arco aórtico do sauim (Saguinus niger) e sua importância no processo de urbanização. Biotemas, Florianópolis, v. 30, n. 4, p. 95-100, 2017.

CULAU, P. O. V.; RECKZIEGEL, S. H.; LINDEMANN, T.; ARAÚJO, A. C. P.; BALZARETTI, F. Colaterais do arco aórtico da capivara (Hidrochoerus hidrochaeris). Acta Scientiae Veterinariae, Porto Alegre, v. 35, p. 89-92, 2007.

FARIA, D. S. O estudo de campo do mico estrela no Planalto Central Brasileiro. In: ADES, C. (Ed.). Etologia de animais e de homens. São Paulo: Edicon/Edusp, 1989. p. 109-121.

FERNANDES FILHO, A. F.; BORELLI, V. Contribuição ao estudo dos colaterais calibrosos do arco aórtico no gato. Revista da Faculdade de Medicina Veterinária de São Paulo, São Paulo, v. 8, n. 2, p. 385-388, 1970.

FURTADO, F. S.; VASCONCELOS, L. D. P.; BRANCO, E.; LIMA, A. R. Anatomia cardíaca e ramificações da aorta em macaco-prego (Sapajus apella). Biotemas, Florianópolis, v. 30, n. 4, p. 83-93, 2017.

INTERNATIONAL COMMITTEE ON VETERINARY GROSS ANATOMICAL NOMENCLATURE. Nomina Anatomica Veterinaria. 6. ed. Knoxville: World Association on Veterinary Anatomist, 2017. 160 p.

ITEREZOTE; A. M.; MEDEIROS, A. D.; BARBOSA FILHO, R. C. C.; PETRELLA, S.; ANDRADE JUNIOR, L. C.; MARQUES, S. R.; PRATES, J. C. Anatomical variation of the brachiocephalic trunk and common carotid artery in neck dissection. International Journal of Morphology, Temuco, v. 27, n. 2, p. 601-603, 2009.

LIMA, A. R.; SOUZA, D. C.; DO CARMO, D. C.; SANTOS, J. T.; BRANCO, E. Ramos colaterais do arco aórtico e suas principais ramificações no cachorro-do-mato (Cerdocyon thous). Pesquisa Veterinária Brasileira, Rio de Janeiro, v. 36, n. 7, p. 647-651, 2016.

MAGALHÃES, M. S.; ALBUQUERQUE, J. F. G.; OLIVEIRA, M. F.; PAPA, P. C.; MOURA, C. E. B. Branches of the aortic arch in mocó (Kerodon rupestris). Revista Portuguesa de Ciências Veterinárias, Lisboa, v. 102, p. 49-52, 2007.

MANSFIELD, K. Marmoset models commonly used in biomedical research. Comparative Medicine, Memphis, v. 53, n. 4, p.383392,2003

MARTINS, D. M.; LIMA, A. R.; PINHEIRO, L. L.; SILVA, S. B. S.; ARAÚJO, E. B.; MELUL, R.; CUNHA, A. C. L.; MENESES, A. M. C.; SOUZA, A. C. B.; PEREIRA, L. C.; FIORETTO, E. T.; BRANCO, E. Morphologic description of the collateral branches from aortic arch and its main ramifications in Leopardus pardalis. Acta Veterinaria Brasilica, Mossoró, v. 4, n. 2, p.74-77, 2010.

MOORE, K. L.; DALLEY, A. F. Anatomia orientada para a clínica. Rio de Janeiro: Guanabara Koogan, 2001. 1023 p.

NITTA, C. Y.; SILVA, L. C. S.; MIGLINO, M. A.; AMBROSIO, C. E.; BOMBONATO, P. P.; RICI, R. E. G. Ultraestrutura comparativa da língua do sagui-de-tufo-preto (Callithrix penicillata) e do bugiopreto (Alouatta caraya) em diferentes faixas etárias. Pesquisa Veterinária Brasileira, Rio de Janeiro, v. 33, n. 1001, p. 75-84, 2013.

NOWAK, R. M. Walker's mammals of the world. Baltimore: The Johns Hopkins University Press, 1999. 836 p. 
OLIVEIRA, F. G.; MACHADO, M. R. F.; MIGLINO, M. A. E.; NOGUEIRA, T. M. Gross anatomical study of the aortic ARC branches of the paca (Agouti paca, Linnaeus, 1766). Brazilian Journal of Veterinary Research and Animal Science, São Paulo, v. 38, n. 3, p. 103-105, 2001.

OLIVEIRA, R. E. M.; OliveirA, G. B.; BARBOSA, P. M. L.; BEZERRA, F. V. F.; GOMES, J. F. A; AMBRÓSIO, C. E.; MIGLINO, M. A.; OLIVEIRA, M. F. Ramos Colaterais do Arco do preá (Galea spixii Wagler, 1831). Pesquisa Veterinária Brasileira, Rio de Janeiro, v. 35, n. 8, p. 762-766, 2015.

PINHEIRO, V. L. C.; LIMA, A. R.; PEREIRA, L. C.; DUARTE, B. G.; BRANCO, E. Descrição anatômica dos ramos colaterais do arco aórtico do tamanduá-mirim (Tamandua tetradactyla). Biotemas, Florianópolis, v. 25, n. 2, p. 133-137, 2012.

RYLANDS, A. B.; MENDES, S. L. A lista vermelha da IUCN de espécies ameaçadas. 2018.2. 2018. Disponível em: <http: www. iucnredlist.org/details/41519/0>. Acesso em: 14 set. 2018.

SANTOS, A. L. Q.; MORAES, F. M.; MALTA, T. S.; CARVALHO, F. S. M.; ALVES JUNIOR, J. R. F. Topografia dos colaterais calibrosos do arco aórtico de um mão-pelada (Proncyon cancrivorus - Gray, 1865), (Carnivora - Proncyoniadae). Archives of Veterinary Science, Curitiba, v. 9, p. 67-72, 2004.

SANTOS, P. R. S.; SILVA, M. H. R.; RODRIGUES, A. R.; ASSIS NETO, A. C. Descrição anatômica do plexo braquial de Callithrix jacchus e Callithrix penicillata. Pesquisa Veterinária Brasileira, Rio de Janeiro, v. 36, n. 9, p. 901-904, 2016.

SCHALlER, O.; NASCIMENTO, F. G. do. Nomenclatura anatômica veterinária ilustrada. São Paulo: Manole, 1999. 614 p. SCHIMMING, B. C.; JESUS, L. S. B. S.; FILADELPHO, A. L. Branching pattern of aortic in the white-eared opossum (Didelphis albiventris). Brazilian Journal of Veterinary Research and Animal Science, São Paulo, v. 53, n. 3, p. 235-242, 2016.

SILVA, K. F. da.; PEREIRA, K. F.; ALBUQUERQUE, K. P.; TEIXEIRA, C. S.; ODA, J. Y. Estudo descritivo das variações anatômicas dos ramos do arco aórtico. Arquivos de Ciências da Saúde da UNIPAR, Umuarama, v. 16, n. 3, p. 101-103, 2012.
SILVA, L. C. S. Contribuição ao estudo do estômago de saguide-tufo-preto (Callithrix penicillata). 2012. 87 f. Dissertação (Mestrado em Ciências) - Universidade de São Paulo, São Paulo. 2012.

SILVA, L. C. S.; BARROSO, C. E.; PRIMO BOMBONATO, P. P. Morfologia do pericárdio do sagui-de-tufo-preto (Callithrix penicillata). Biotemas, Florianópolis, v. 25, n. 1, p. 131-135, $2012 \mathrm{a}$.

SILVA, L. C. S.; LESSA, T. B.; SILVA SANTO, P. R.; GRASSI RIC, R. E.; BOMBONATO, P. P.; AMBRÓSIO, C. E. Arquitetura da árvore brônquica no sagui-de-tufo-preto: um modelo animal experimental para lesões do sistema respiratório. Archives of Veterinary Science, Curitiba, v. 17, n. 4, p. 63-69, 2012 b.

SILVA, M. O. M.; DE ARMADA, J. L. A.; VERONA, C. E. S.; HELIODORO, G.; NOGUEIRA, D. M. Cytogenetics and molecular genetic analysis of chimerism in marmosets (Callithrix: Primates). Anais da Academia Brasileira de Ciências, Rio de Janeiro, v. 89, n. 4, p. 2793-2804, 2017.

SOUZA, F.; ZECHIN, A. B.; CAMPOS, R. Ramos colaterais do arco aórtico e suas principais ramificações em coelho da raça Nova Zelândia (Oryctolagus cuniculus). Ciência Rural, Santa Maria, v. 43, n. 12, p. 2261-2267, 2013.

STEVENSON, M. F.; RYLANDS, A. B. The marmoset, genus Callithrix. In: MITTERMEIER, R. A.; RYLANDS, A. B.; COIMBRA-FILHO, A.; FONSECA, G. A. B. (Ed.). Ecology and behaviour of Neotropical Primates. 2. ed. Washington: World Wild Life Fundation, 1988. p. 131-222.

TORTORA, G. J. Princípios de anatomia humana. Rio de Janeiro: Guanabara Koogan, 2007. 1017 p.

VALLE, R. R. Colheita, análise e criopreservação de sêmen de uma espécie modelo de primata neotropical. Sagui-de-tufobranco (Callithrix jacchus). 2007. 169 f. Tese (Doutorado em Medicina Veterinária) - Universidade de São Paulo, São Paulo. 2007. 